\title{
Optimizing the performance of non-fading and fading networks using CSMA with joint transmitter and receiver sensing
}

\author{
Mariam Kaynia* and Geir E Øien
}

\begin{abstract}
We consider a mobile ad hoc network where packets belonging to specific transmitters arrive randomly in space and time according to a 3-D Poisson point process, and are upon arrival transmitted to their intended destinations using the carrier sensing multiple access (CSMA) MAC protocol. A packet transmission is considered successful if the received SINR is above a predefined threshold for the duration of the packet. A simple fully-distributed joint transmitter-receiver sensing scheme is proposed for the CSMA protocol to improve its performance in both non-fading and fading networks. The outage probability of this enhanced version of CSMA is derived and optimized with respect to the sensing thresholds. In order to derive a mathematical expression for the optimal sensing thresholds, the inherent hidden and exposed node problems of CSMA are considered and efficiently balanced. The performance of this improved CSMA protocol is compared to the other flavors of CSMA, and shown to bring about significant performance gain.
\end{abstract}

\section{Introduction}

Medium access control (MAC) layer design is commonly applied to address the problem of allocating scarce resources in a wireless network. Various MAC protocols are proposed in order to share the communication channel in the most efficient manner, in order to minimize the destructive interference. One such protocol that has gained great popularity is carrier sensing multiple access (CSMA). This protocol is successfully employed in the IEEE 802.11 standard family, and many enhancements have been proposed and implemented in order to minimize the inherent hidden and exposed node problems [1-3]. Nevertheless, there is still room for improvement, in particular in networks with high density of simultaneous transmissions. This provides the motivation behind our study.

Numerous studies have evaluated the performance of the CSMA protocol, evaluating its performance in terms of throughput and bit error rate [4-6]. Much of this study confirms CSMA's superiority over other protocols, such as ALOHA, with natural tradeoffs in other domains such

\footnotetext{
*Correspondence: mariam.kaynia@iet.ntnu.no

Department of Electronics and Telecommunications, Norwegian University of Science and Technology, 7491 Trondheim, Norway
}

as transmission rate and delay $[1,6]$. Also, many extensions have been proposed to improve the performance of CSMA [7-9]. However, the conventional model used in most of these studies assumes that the topology of the network is known, and that multiple links cannot communicate simultaneously. Such assumptions are not realistic for mobile ad hoc networks (MANETs). The closest model to a real-life ad hoc network is that used in $[10,11]$, where users are assumed to be Poisson distributed in space and each transmitter communicates with its own receiver using ALOHA, while allowing for simultaneous communication between links. This model was embraced in [12], and is also applied in our study.

Choosing the right value for CSMA's sensing threshold (which the backoff decision is based on) is of great importance for the performance of this protocol. Many studies have proposed various adaptation schemes to find the optimal carrier sensing threshold of CSMA to enhance the throughput and the transmission reliability in dynamically changing networks [13-15]. In [2], a novel analytical model was introduced for determining the optimal carrier sensing range in ad hoc networks by minimizing the sum of the hidden and exposed terminal areas. The optimization is done in terms of aggregate throughput, 
yielding that the optimal carrier sensing range is approximately equal to the interference range. The study of [16] further improves the carrier sensing capability of CSMA by adding fairness to the equation, while retaining the throughput performance. The shortcoming of this study is that only six node pairs are considered in the performance evaluation, limiting the applicability of the results to a randomly distributed ad hoc network with high density of simultaneous transmissions. Moreover, it is shown in [7] that the optimal algorithm is for the senders to keep the product of their transmit power and carrier sensing threshold equal to a constant. However, this algorithm is not distributed, and is dependent on the estimation of signal powers. Another algorithm for maximizing the throughput is to decrease the sensing range as long as the network remains sufficiently connected [17]. An improved carrier sensing threshold adaptation algorithm was proposed in [8], where each node chooses the sensing threshold that maximizes the number of successful transmissions in its neighborhood. The drawback of this technique is that it relies on the collection of information over a period of time, which entails higher complexity, and introduces delays. Zhu et al. derive in [9] the optimal sensing threshold of the conventional CSMA protocol to be $\beta_{t}=\rho\left(1+\beta^{1 / \alpha}\right)^{\alpha}$, where $\rho$ represents the transmitted signal strength, $\alpha$ is the path loss exponent, and $\beta$ is the minimum required signal-to-interference-plus-noise ratio (SINR) threshold for correct reception of packets. The optimized CSMA protocol was evaluated on a real test-bed in [18].

In [12], a new version of the CSMA protocol is proposed, denoted CSMA $A_{R X}$, where the receiver (as opposed to the transmitter in $\mathrm{CSMA}_{\mathrm{TX}}$ ) performs the channel sensing and makes the backoff decision. The sensing thresholds of both CSMA $\mathrm{TX}_{\mathrm{TX}}$ and $\mathrm{CSMA}_{\mathrm{RX}}$ are optimized in a non-fading ad hoc network. It is shown that for lower densities, applying a sensing threshold may not provide any improvement compared to having no sensing at all. For higher densities, however, significant reduction in the outage probability can be obtained by setting the sensing threshold of the transmitter in $\mathrm{CSMA}_{\mathrm{TX}}, \beta_{t}$, or of the receiver in $\mathrm{CSMA}_{\mathrm{RX}}, \beta_{r}$, equal to the communication threshold, $\beta$, i.e., $\beta_{t}^{\text {opt }} \approx \beta_{r}^{\text {opt }} \approx \beta$.

Having considered the performance of CSMA $_{\text {TX }}$ and $\mathrm{CSMA}_{\mathrm{RX}}$, a natural question then becomes: Can we improve the performance of CSMA further if we allow both the transmitter and its receiver to sense the channel, and subsequently let them collectively decide whether or not to initiate transmission of each packet? And moreover, what are the optimal sensing thresholds that minimize the outage probability of this new flavor of CSMA both in the absence and presence of fading?

Hence, in the following, we will analyze the impact of a joint backoff decision making on the performance of the CSMA protocol. Following the same style of notation as in [12], we refer to this modified flavor of CSMA as CSMA TXRX. The analytical framework used in the present study is inspired from [12], with the addition of allowing for a joint backoff decision making mechanism, and adding fading effects to the network model. The concept of a joint backoff decision making was first introduced in [19], where only a non-fading network was considered, and the MAC protocols did not allow for multiple backoffs and retransmissions, something that simplified the analysis significantly. Not only is the analysis of the present article useful for future improvements made to CSMA, it also provides us with a fundamental understanding of the hidden and exposed node problems, which are the main sources of imperfection of this protocol. This in-depth understanding is used to derive the optimal sensing thresholds of CSMA TXRX, $_{\text {, }}$ something that would otherwise be too complicated to achieve. The hidden node problem occurs whenever a new node is unable to detect an ongoing transmission, so that it initiates its transmission and thereby causes outage for an already active packet. The exposed node problem is characterized by transmissions being prevented even though they could have taken place without harm to other ongoing transmissions. A decrease in one of these two problems, results in an increase in the other, and vice versa. Choosing optimal values for the sensing thresholds $\beta_{t}$ and $\beta_{r}$ will provide a balance between the hidden and exposed node problems, thus improving the network performance.

\section{System model}

Our network model considers a mobile network where packets are distributed randomly in space and time according to a 3-D Poisson point process (PPP). At a snapshot in time, the network consists of transmitter-receiver pairs distributed randomly on a 2-D plane according to a homogeneous spatial PPP with density $\lambda^{s}$ [nodes $/ \mathrm{m}^{2}$ ]. At each transmitter, a series of packets, each with a fixed duration $T$, arrives according to an independent 1-D PPP in time with intensity $\lambda^{t}$ [packets/sec/node]. If we only consider the arrival of new packets in space, the packet density is $\lambda=\lambda^{s} \lambda^{t} T$. As this network model entails two independent Poisson distributions, we would have to average over both the spatial and temporal statistics to obtain the outage probability, making the mathematical analysis infeasible. Thus, we consider our wireless network from an alternative point of view: Upon the arrival of each packet, it is assigned to a transmitter node, which is then randomly placed on a 2-D plane (uniformly distributed in area $A$ ), as illustrated in Figure 1. Upon the formation of each packet and according to the specified MAC protocol, it is transmitted with constant power $\rho$ 
to its intended receiver a fixed distance $R$ away. When the packet has been served (successfully or not), the corresponding transmitter-receiver pair disappears from the plane. As long as the maximum number of backoffs, $M$, and retransmissions, $N$, is not reached, the packet is placed back in the packet arrival queue, with a new transmission time. The retransmitted packet will be located in a new position, which is justified by our assumption of high mobility.

Such a 3-D representation of the network model simplifies our study, as it allows us to consider a single random process describing both the temporal and spatial variations of the system, something that facilitates analyses that previously have been perceived as too complicated to perform. Note that the temporal PPP of packet arrivals at each node is independent of the PPP of transmitter locations in space. Due to the high mobility assumption in our network, different sets of packets are active between times $t_{0}$ and $t_{0}+T$. Since the waiting time from one transmission attempt to the next is set to be greater than $T$, we have that there are no spatial and temporal correlations between retransmission attempts. Moreover, note that the fixed distance of $R$ between each transmitterreceiver pair does not affect the Poisson distribution of the interferers. That is, from the point of view of each node (receiver or transmitter), the interfering transmitters have a random topology following a PPP, meaning that many of the interfering transmitters could be at a random distance less than $R$ away. Also, setting the distance between each transmitter-receiver pair to a fixed value, does not impact our analysis and results, as we are considering the lower bound to the outage probability. To prove this claim, consider the general expression for the probability of an erroneous packet reception in an interference-limited PPP network, where $d$ denoting the

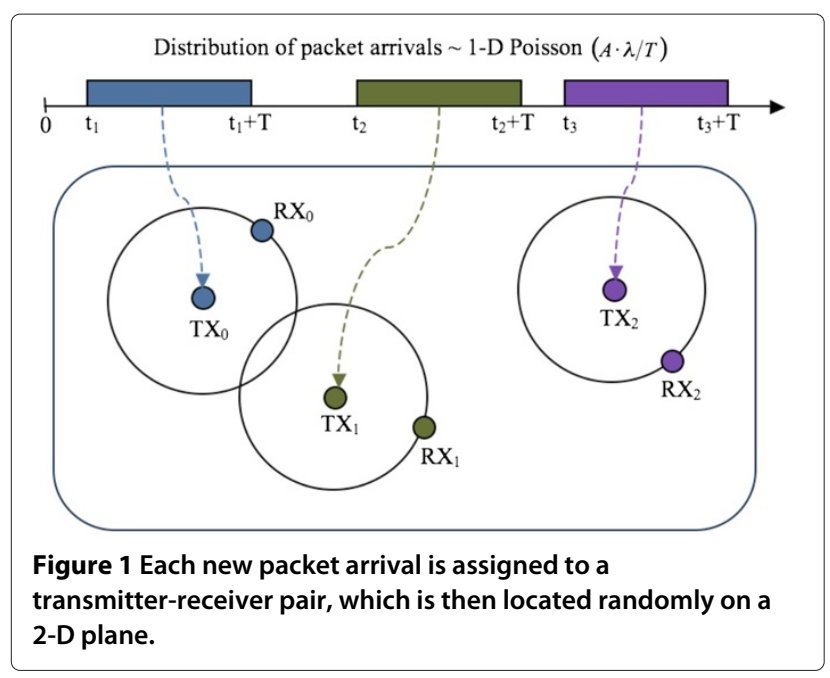

distance between a transmitter and its own receiver, is a random variable:

$$
\begin{aligned}
P_{\text {error }}(d) & =\operatorname{Pr}[\operatorname{SINR} \leq \beta] \\
& \geq \mathbb{E}_{d}\left[1-e^{-k d^{2}}\right] \quad ; \text { where } k=\lambda \pi \beta^{-2 / \alpha} \\
& \approx 1-e^{-k \mathbb{E}_{d}\left[d^{2}\right]} \\
& \geq 1-e^{-k \mathbb{E}_{d}[d]^{2}},
\end{aligned}
$$

where we have used: $\mathbb{E}_{d}\left[d^{2}\right]=\operatorname{var}(d)+\mathbb{E}_{d}[d]^{2}$. Replacing $\mathbb{E}_{d}[d]$ by a fixed value $R$ yields the error expression we use in our analyses and thereby confirms that our assumption on the fixed transmitter-receiver distance does not impact the lower bound outage probability analysis significantly. Also, it is worthwhile noting that the whole network with a fixed $R$ could be viewed as a snapshot of a multi-hop wireless network, where $R$ is the bounded average interrelay distance. For more details on this 3-D model, please refer to [12].

Our traffic model has the following main attributes, which will be significant in our derivations;

- Our network is highly mobile, meaning that different and independent sets of nodes are observed on the plane from one slot (of duration $T$ ) to the next.

- The waiting time between each retransmission attempt, $t_{\text {wait }}$, is by design ensured to be more than $T$. Because of the high mobility assumption, new channel instances are observed between transmission attempts, and thus there are no temporal correlations between retransmissions.

- Upon retransmission of a packet, it is treated as a new packet arrival and placed in a new location, resulting are no spatial correlations between retransmission attempts.

For the channel model, we consider both non-fading and fading networks. In the former, only path loss attenuation effects are considered, with path loss exponent $\alpha>2$. Each receiver potentially sees interference from all transmitters, and these independent interference powers are added to the channel noise $\eta$ to cause signal degradation. The introduction of fading adds a new source of randomness to the model, namely the channel coefficients $h_{i j}$. The SINR in a non-fading network and the $\operatorname{SINR}^{f}$ in a fading network are given respectively as

$$
\operatorname{SINR}=\frac{\rho R^{-\alpha}}{\eta+\sum_{i(t)} \rho r_{i}^{-\alpha}} \wedge \operatorname{SINR}^{f}=\frac{\rho R^{-\alpha} h_{00}}{\eta+\sum_{i(t)} \rho r_{i}^{-\alpha} h_{0 i}}
$$

where $r_{i}$ is the distance between the node under observation and the $i$ th interfering transmitter; $h_{00}$ represents the fading effects between the receiver under observation, $\mathrm{RX}_{0}$, and its designated transmitter; $h_{0 i}$ is the fading coefficient between $\mathrm{RX}_{0}$ and the $i$ th interfering transmitter. 
The summation is over all active interferers on the plane at a given time instant $t$.

\subsection{MAC protocol}

The channel access is driven by the CSMA $\mathrm{AXRX}_{\text {TX }}$ protocol. This protocol operates as follows: When the transmitter has a packet to transmit, it performs physical carrier sensing of the interference power, $P_{\mathrm{int}}$, in the channel (i.e., the radio measures the energy received on its available radio channel). Based on this measurement, and its knowledge on the distance $R$ to its own receiver, the transmitter calculates the expected received SINR by using Equation (2), where the denominator is replaced by the measured $P_{\text {int }}$. If this expected received SINR is below the required threshold $\beta_{t}$, the channel is considered busy and the transmitter refrains from transmission. If it is above $\beta_{t}$, a request-to-send (RTS) signal is sent to the receiver, which then performs a similar channel sensing of the interference power around itself. If this measured SINR at the receiver is below the required sensing threshold $\beta_{r}$, it informs its transmitter to cancel the transmission; if the measured SINR is above $\beta_{r}$, a clear-to-send (CTS) signal is sent to the transmitter, which then initiates the packet transmission.

Once a transmission is initiated, there is still a probability that the packet is received in error at its destination, i.e., the received SINR is below $\beta$ at some $t \in(0, T)$. In this case, the packet is retransmitted. Each packet is given $M$ backoffs and $N$ retransmissions before it is dropped and counted to be in outage.

Note that the main difference between the proposed

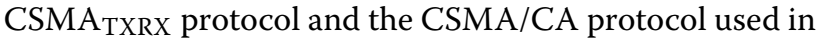
the IEEE 802.11 standard is that in the latter, all nodes hear the RTS and CTS signals, whereas in CSMA TXRX, the communication of control signals occurs between a transmitter and its own receiver only (e.g., by using coding or separate frequency bands). Such isolated signaling scheme has a few benefits: (a) collision between the control signals is avoided, (b) delays in the decision-making stage are reduced, and (c) the situation where nodes unnecessarily decide to back off due to the detected RTS/CTS signal (even though their packets would have been received correctly at their own receivers) is mitigated.

\subsection{Performance metric}

Our performance metric is outage probability, which is defined as the probability that a packet is received erroneously at its receiver after $M$ backoffs and $N$ retransmissions. Outage probability is closely related to the ubiquitous metric Transmission Capacity, given as

$$
\mathcal{S}=\lambda b\left(1-P_{\text {out }}\right)
$$

where $b$ is the average rate that a successful packet achieves, with units [bits/s/Hz per packet]. The unit of $\mathcal{S}$ is [bits $\left./ \mathrm{s} / \mathrm{Hz} / \mathrm{m}^{2}\right]$. In the following sections, we derive the outage probability of CSMA TXRX $_{\text {both in the absence and }}$ in the presence of fading. In Section 5 , the sensing threshold of the transmitter, $\beta_{t}$, and that of the receiver, $\beta_{r}$, are both optimized.

\section{Performance in the absence of fading}

In this section, we assume no fading effects in the channel, i.e., the signal degradation is due to path loss only, as described in Section 2. First, we explain the method of analysis as well as the key steps of the mathematical derivations, before the result of the analysis is presented in Subsection 3.2.

\subsection{Method of analysis}

Denoting the SINR based on the transmitter's sensing by $\mathrm{SINR}_{t}$, and that based on the receiver's sensing by $\mathrm{SINR}_{r}$, the outage probability of CSMA TXRX is given as

$$
\begin{gathered}
P_{\text {out }}\left(\text { CSMA }_{\text {TXRX }}\right)= \\
\text { Pr }\left[\operatorname{SINR}_{t} \text { and } \text { SINR }_{r}\right. \text { below thresholds } \\
\text { during } M \text { backoffs and } N+1 \\
\text { retransmissions }] \\
=\operatorname{Pr}\left[\operatorname{SINR}_{t}<\beta_{t} \cup \operatorname{SINR}_{r}<\beta_{r} \text { at } t=0\right]^{M} \\
-\left(1-\operatorname{Pr}\left[\operatorname{SINR}_{t}<\beta_{t} \cup \operatorname{SINR}_{r}\right.\right. \\
\left.\left.<\beta_{r} \text { at } t=0\right]^{M}\right) \\
\operatorname{Pr}\left[\operatorname{SINR}_{r}<\beta \text { at some } t \in[0, T)\right]^{N+1} .
\end{gathered}
$$

Due to the distance dependence of the interference, in order to derive expressions for the above terms, we apply the concept of guard zones [10]. Defining $s_{r}$ to be the distance between the receiver under observation, $\mathrm{RX}_{0}$, and its closest interferer, $\mathrm{TX}_{i}$, that causes the SINR to fall just below the threshold $\beta_{r}$, yields

$$
s_{r}=\left(\frac{R^{-\alpha}}{\beta_{r}}-\frac{\eta}{\rho}\right)^{-\frac{1}{\alpha}} .
$$

Based on Equation (5), $\beta_{t}$ corresponds to $s_{t}$ and $\beta$ to $s$. Denote the circle of radius $s_{r}$ around $\mathrm{RX}_{0}$ by $B\left(\mathrm{RX}_{0}, s_{r}\right)$, and the circle of radius $s_{t}$ around $\mathrm{TX}_{0}$ by $B\left(\mathrm{TX}_{0}, s_{t}\right)$. Following Equation (4) and the concept of guard zones, we have that if there exists at least one transmission inside $B_{1}=B\left(\mathrm{TX}_{0}, s_{t}\right) \cup B\left(\mathrm{RX}_{0}, s_{r}\right)$ upon the arrival of $\mathrm{TX}_{0}-\mathrm{RX}_{0}$, this transmitter-receiver pair would back off from transmission. The area of $B_{1}$ is shown as the lightly shaded area in Figure 2, and is given as $\pi s_{t}^{2}+\pi s_{r}^{2}-A_{o l}\left(s_{t}, s_{r}\right)$, where $A_{o l}\left(s_{t}, s_{r}\right)$ denotes the area of overlap $B\left(\mathrm{TX}_{0}, s_{t}\right) \cap$ $B\left(\mathrm{RX}_{0}, s_{r}\right)$, and is simply derived to be 


$$
A_{o l}\left(s_{t}, s_{r}\right)= \begin{cases}0 ; & s_{r}+s_{t}<R \\ \pi s_{t}^{2} ; & s_{r}>R+s_{t} \\ \pi s_{r}^{2} ; & s_{t}>R+s_{r} \\ s_{r}^{2} \cos ^{-1}\left(\frac{R^{2}+s_{r}^{2}-s_{t}^{2}}{2 R s_{r}}\right)+s_{t}^{2} \cos ^{-1}\left(\frac{R^{2}+s_{t}^{2}-s_{r}^{2}}{2 R s_{t}}\right) & \\ & \\ & \quad-\frac{1}{2} \sqrt{\left(s_{t}+s_{r}-R\right)\left(s_{t}-s_{r}+R\right)\left(-s_{t}+s_{r}+R\right)\left(s_{t}+s_{r}+R\right)} ;\end{cases}
$$

Once $\mathrm{TX}_{0}$ and $\mathrm{RX}_{0}$ jointly decide to transmit, there is still a probability that their packet is in error upon its arrival due to an ongoing transmission inside $B\left(\mathrm{RX}_{0}, s\right)$ that was not detected in the backoff decision-making stage. That is, the packet is in error at the start, if an active transmission is detected inside $B\left(\mathrm{RX}_{0}, s\right) \cap$ $\overline{B\left(\mathrm{TX}_{0}, s_{t}\right) \cup B\left(\mathrm{RX}_{0}, s_{r}\right)}$. This area is marked as the darkly shaded region in Figure 2, and is given by

$A_{r x \mid \text { active }}\left(s_{t}, s_{r}, s\right)= \begin{cases}0 ; & s_{r} \geq s \\ \pi s^{2}-\pi s_{r}^{2}-A_{o l}\left(s_{t}, s\right) ; & s_{r}<s \text { and } s_{t}<R-s_{r} \\ \pi s^{2}-\pi s_{r}^{2}-A_{o l}\left(s_{t}, s\right) & \\ +A_{o l}\left(s_{t}, s_{r}\right) ; & s_{r}<s \text { and } s_{t}<R+s_{r} \\ \pi s^{2}-A_{o l}\left(s_{t}, s\right) ; & \text { otherwise }\end{cases}$

Now, given the packet transmission of $\mathrm{TX}_{0}-\mathrm{RX}_{0}$ is initiated and it is not in error at the start of its transmission, there is a probability that a new interferer, $\mathrm{TX}_{i}$, enters the plane at some $t \in(0, T)$, is located inside $B\left(\mathrm{RX}_{0}, s\right)$, and thus causes error for the packet of $\mathrm{RX}_{0}$. Since $\mathrm{TX}_{i}$ would back off if it or its receiver detect the transmission of $\mathrm{TX}_{0}$, this means that in order for $\mathrm{TX}_{i}$ to cause outage for $\mathrm{RX}_{0}$, it must be placed inside $B_{2}=B\left(\mathrm{RX}_{0}, s\right) \cap \overline{B\left(\mathrm{TX}_{0}, s_{t}\right)}$, while its receiver $\mathrm{RX}_{i}$ is located outside of $B\left(\mathrm{TX}_{0}, s_{r}\right)$. This probability is denoted as $P_{\text {during, }}$, and is given in the following subsection.

Due to the Poisson distribution of interferers, we apply the expression $P_{\text {error }}=1-\exp \left\{-\lambda_{\text {csma }} A\right\}$, where $A$ is the de-

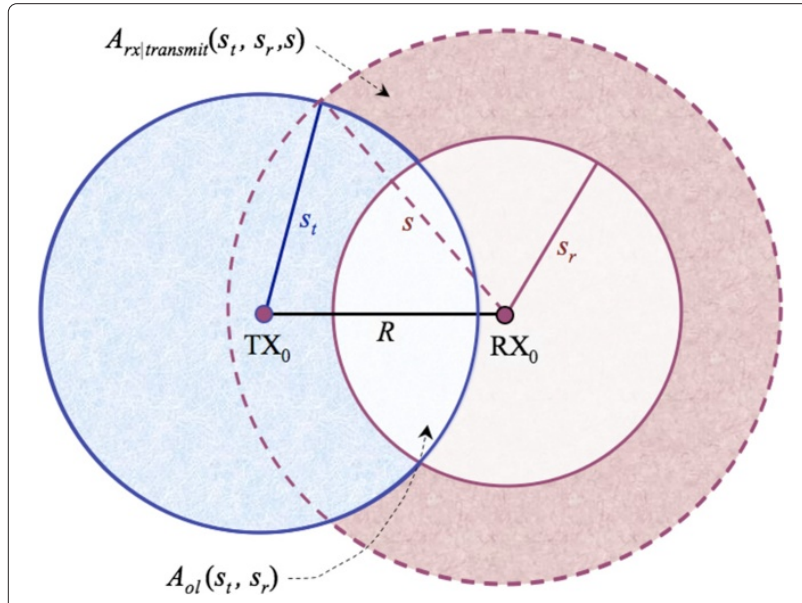

Figure 2 Geometrical illustration of sensing zones $B\left(\mathbf{R X}_{0}, s_{r}\right)$ and $B\left(\mathrm{TX}_{0}, s_{t}\right)$. tection area depending on the particular error probability to be calculated. Moreover, the density $\lambda_{\text {csma }}$ is given as

$$
\begin{aligned}
\lambda_{\text {csma }} & = \begin{cases}\lambda \sum_{m=0}^{M-1} P_{b}^{m} ; & \text { for } N=0 \\
\lambda\left[\sum_{m=0}^{M-1} P_{b}^{m}+\left(1-P_{b}^{M}\right) P_{r t 1} \sum_{n=0}^{N-1} P_{r t}^{n}\right] ; \text { for } N \geq 1\end{cases} \\
& =\lambda\left[\frac{1-P_{b}^{M}}{1-P_{b}}+\left(1-P_{b}^{M}\right) P_{r t 1} \frac{1-P_{r t}^{N}}{1-P_{r t}}\right],
\end{aligned}
$$

where $P_{b}$ is the backoff probability, $P_{r t 1}$ is the probability that the packet is received in error at its first transmission attempt, and $P_{r t}$ is the probability that the packet is received erroneously in a retransmission attempt. The reason we distinguish between $P_{r t 1}$ and $P_{r t}$ is that due to the backoff decision making stage, the area where there is a probability of detecting an active interferer during a transmission is smaller in the first transmission than in the following retransmissions.

\subsection{Outage probability in non-fading networks}

Based on the above derivations, we are now able to mathematically express the outage probability of CSMA TXRX in a non-fading network. This is given by the following theorem.

Theorem 1. The outage probability of CSMA $A_{T X R X}$ in the absence of fading with varying sensing thresholds is given by

$$
P_{\text {out }}\left(C S M A_{T X R X}\right)=P_{b}^{M}+\left(1-P_{b}^{M}\right) P_{r t 1} P_{r t}^{N},
$$

where:

- $P_{b}$ is the backoff probability, approximated by the solution to

$$
\tilde{P}_{b}=1-e^{-\lambda\left[1-\tilde{P}_{b}^{M}+\left(1-\tilde{P}_{b}^{M}\right) \tilde{P}_{r t 1} \frac{1-\tilde{P}_{r t}^{N}}{1-\tilde{P}_{r t}}\right]\left(\pi s_{t}^{2}+\pi s_{r}^{2}-A_{o l}\left(s_{t}, s_{r}\right)\right)}
$$

where $A_{o l}\left(s_{t}, s_{r}\right)$ is given by Equation (6).

- $P_{r t}=P_{r x}+\left(1-P_{r x}\right) P_{\text {during }}$ is the probability that a packet is received in error in a retransmission attempt. $P_{r x}$ is the probability that the packet is in error at the start of each of its retransmissions, approximated by

$$
\tilde{P}_{r x}=1-e^{-\lambda\left[1-\tilde{P}_{b}^{M}+\left(1-\tilde{P}_{b}^{M}\right) \tilde{P}_{r t 1} \frac{1-\tilde{P}_{r t}^{N}}{1-\tilde{P}_{r t}}\right] \pi s^{2}},
$$

- $P_{\text {during }}$ is the probability that an error occurs at some $t \in(0, T)$, approximated by

$$
\tilde{P}_{\text {during }}=1-e^{-\lambda\left[\frac{1-\tilde{P}_{b}^{M}}{1-\tilde{P}_{b}}+\left(1-\tilde{P}_{b}^{M}\right) \tilde{P}_{r t 1} \frac{1-\tilde{P}_{r t}^{N}}{1-\tilde{P}_{r t}}\right] G_{\text {active }}\left(s_{t}, s_{r}\right)}
$$


where $G_{\text {active }}\left(s_{t}, s_{r}\right)$ is given as

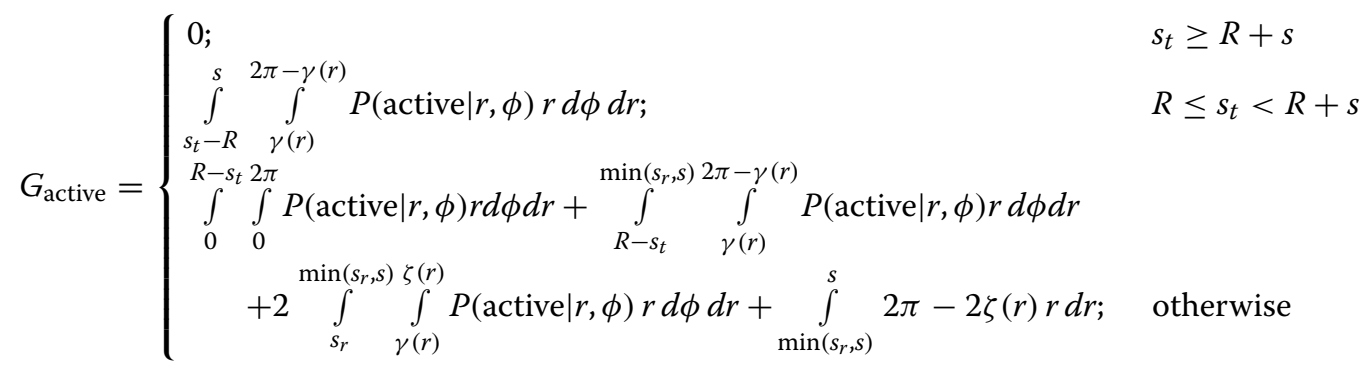

$\nu(r)$ and $P($ active $\mid r, \phi)$, which is the probability that the $R X_{i}$ is not in error upon its arrival, are given as

$P($ active $\mid r, \phi)=1-\frac{1}{\pi} \cos ^{-1}\left(\frac{r^{2}+2 R^{2}-s_{r}^{2}-2 R r \cos \phi}{2 R \sqrt{r^{2}+R^{2}-2 R r \cos \phi}}\right)$

$$
\begin{aligned}
v(r) & =\cos ^{-1}\left(\frac{r^{2}+2 R s_{t}-s_{t}^{2}}{2 R r}\right) \wedge \zeta(r) \\
& =\cos ^{-1}\left(\frac{r^{2}-2 R s_{t}-s_{t}^{2}}{2 R r}\right) \wedge \gamma(r) \\
& =\cos ^{-1}\left(\frac{r^{2}+R^{2}-s_{t}^{2}}{2 R r}\right) .
\end{aligned}
$$

- $P_{r t 1}=P_{r x \mid \text { active }}+\left(1-P_{r x \mid \text { active }}\right) P_{\text {during }}$ is the probability that the packet is received in error at its first transmission attempt. $P_{r x \mid \text { active }}$ is the probability that the receiver is in outage at the start of the packet, although it decides to initiate its transmission, approximated by

$$
\tilde{P}_{r x \mid \text { active }}=\tilde{P}_{r x} \frac{A_{r x \mid \text { active }}\left(s_{t}, s_{r}, s\right)}{\pi s^{2}},
$$

with $A_{r x \mid \text { active }}\left(s_{t}, s_{r}, s\right)$ given by Equation (7).

For more details on the derivation of Equations (10) and (14), please refer to [12]. Optimization of the sensing thresholds is carried out in Section 5, and comparison between CSMA TXRX with the other CSMA versions is performed in Section 6.

\section{Performance in the presence of fading}

In this section, we add fading effects to the path loss attenuation, as described in Section 2. Due to the independence of the channel fading coefficients on distance, we can no longer operate with the closest interferer for the derivation of the outage probability. Instead, we must consider the dominant interferer, which is a single interferer whose received interference power (affected by the distance and the random channel coefficients) alone is strong enough to result in outage for the packet under observation.
Similar to Section 3, we first explain the method of analysis as well as the key steps of the mathematical derivations, before the result of the analysis is presented in Subsection 4.2.

\subsection{Method of analysis}

The outage probability expression in the case of fading is the same as Equation (9), with the difference that $P_{b}, P_{r t 1}$, and $P_{r t}$ are replaced by their average values with respect to the fading coefficients, namely $\bar{P}_{b}, \bar{P}_{r t 1}$, and $\bar{P}_{r t}$. Based on the same reasoning as in the non-fading case in Section 3 , the density of packets attempting to access the channel is

$$
\lambda_{\mathrm{csma}}=\lambda\left[\frac{1-\bar{P}_{b}^{M}}{1-\bar{P}_{b}}+\left(1-\bar{P}_{b}^{M}\right) \bar{P}_{r t 1} \frac{1-\bar{P}_{r t}^{N}}{1-\bar{P}_{r t}}\right] .
$$

The probability that a given transmitter-receiver pair, $\mathrm{TX}_{0}-\mathrm{RX}_{0}$, backs off is given by the probability that the SINR at the start of the packet is below $\beta_{t}$ at the transmitter, or below $\beta_{r}$ at the receiver, or both. Hence,

$$
\bar{P}_{b}=\bar{P}_{b 1}\left(\beta_{t}\right)+\bar{P}_{b 1}\left(\beta_{r}\right)-\operatorname{Pr}\left(\mathrm{TX}_{0} \text { beg. } \cap \mathrm{RX}_{0} \text { beg. }\right)
$$

$\bar{P}_{b 1}\left(\beta_{t}\right)$ and $\bar{P}_{b 1}\left(\beta_{r}\right)$ are derived by using a modified version of the guard zone concept discussed in Section 3. With an SINR constraint of $\beta \in\left(\beta_{t}, \beta_{r}\right)$, define the distance to the dominant interferer (given $h_{00}$ and $h_{0 i}$ ) as

$$
s^{f}\left(h_{00}, h_{0 i}\right)=h_{0 i}^{1 / \alpha}\left(\frac{h_{00} R^{-\alpha}}{\beta}-\frac{\eta}{\rho}\right)^{-1 / \alpha} .
$$

Consequently, we have that the average number of dominant interferers within a distance $s^{f}\left(h_{00}, h_{0 i}\right)$ away and with arrival time during $(-T, 0)$ is approximated by $\pi \lambda_{\text {csma }} s^{f}\left(h_{00}, h_{0 i}\right)^{2}$. Due to the Poisson distribution of packets, the backoff probability then becomes

$$
\begin{aligned}
\bar{P}_{b 1}(\beta) & \geq 1-\mathbb{E}\left[e^{-\pi \lambda_{\mathrm{csma}} s^{f}\left(h_{00}, h_{0 i}\right)^{2}}\right] \\
& \approx 1-\exp \left\{-\pi \lambda_{\mathrm{csma}} \mathbb{E}\left[s^{f}\left(h_{00}, h_{0 i}\right)^{2}\right]\right\} .
\end{aligned}
$$


Assuming a strictly interference-limited network (i.e., $\eta \approx 0$ ) with Poisson distributed packets, we have

$\bar{P}_{b 1}(\beta) \approx 1-\exp \left\{-\pi \lambda_{\text {active }} \mathbb{E}\left[h_{0 i}^{2 / \alpha}\right] \mathbb{E}\left[\left(\frac{h_{00} R^{-\alpha}}{\beta}\right)^{-2 / \alpha}\right]\right\}$,

where $\lambda_{\text {active }}=\lambda\left[1-\bar{P}_{b}^{M}+\left(1-\bar{P}_{b}^{M}\right) \bar{P}_{r t 1} \frac{1-\bar{P}_{r t}^{N}}{1-\bar{P}_{r t}}\right]$. Note that in order to find $\lambda_{\text {active }}$ only the first term of Equation (8) is multiplied by $\left(1-\bar{P}_{b}\right)$, because once a packet transmission is initiated (the second term in Equation (8)), no further decision-making is performed for each retransmission attempt. Furthermore, knowing that for Rayleigh fading channels, $\mathbb{E}\left[h_{00}^{-2 / \alpha}\right]$ and $\mathbb{E}\left[h_{0 i}^{2 / \alpha}\right]$ are Gamma functions, we may apply the result [20]

$$
\mathbb{E}\left[h_{00}^{-2 / \alpha}\right] \mathbb{E}\left[h_{0 i}^{2 / \alpha}\right]=\frac{2 \pi / \alpha}{\sin (2 \pi / \alpha)},
$$

which is inserted back into Equation (20).

To derive the backoff probability, we return to our geometrical analysis again. We assume that $B\left(\mathrm{TX}_{0}, s_{t}^{f}\right)$ and $B\left(\mathrm{RX}_{0}, s_{r}\right)$ are approximately circular regions, as shown in Figure 2. With $\bar{P}_{b 1}(\beta)$ given by Equation (19), $\operatorname{Pr}\left(\mathrm{RX}_{0}\right.$ beg. $\cap \mathrm{TX}_{0}$ beg. $)$ is derived to be

$$
\begin{aligned}
\operatorname{Pr}\left(\mathrm{RX}_{0} \text { beg. } \cap \mathrm{TX}_{0} \text { beg. }\right)= & \operatorname{Pr}\left(\mathrm{RX}_{0} \text { beg. }\right)-\operatorname{Pr}\left(\overline{\mathrm{TX}_{0} \text { beg. }}\right) \\
& \times \operatorname{Pr}\left(\mathrm{RX}_{0} \text { beg. } \mid \overline{\mathrm{TX}} \text { beg. }\right) \\
= & \bar{P}_{b 1}\left(\beta_{r}\right)-\left[1-\bar{P}_{b 1}\left(\beta_{t}\right)\right] \\
& \times \bar{P}_{b 1}\left(\beta_{r}\right) \frac{\pi s_{r}^{f^{2}}-A_{o l}\left(s_{t}^{f}, s_{r}^{f}\right)}{\pi s_{r}^{2}} .
\end{aligned}
$$

Inserting this expression back into Equation (17), yields the backoff probability of CSMA TXRX. $_{\text {. }}$

Once a transmission has been initiated, there is a probability that the packet is in error at the start of its first transmission attempt. This is denoted by $P_{r x \mid \text { active }}\left(h_{00}, h_{0 i}\right)$. Using geometry again, this probability is given as the probability that an active interferer already exist on the plane inside $B\left(\mathrm{RX}_{0}, s^{f}\right)$, that was not detected during the backoff decision-making stage. That is, the interferer $\mathrm{TX}_{i}$ must be located inside $A_{r x \mid \text { active }}\left(s_{t}^{f}, s_{r}^{f}, s^{f}\right)$, which is shown as the darkly shaded area in Figure 2 and is approximated by Equation (7).

To derive the probability that a packet is received in error at some time during its transmission, denoted by $\bar{P}_{\text {during, we use the Poissonianity of interferers and apply }}$ the following expression:

$$
\bar{P}_{\text {during }}=\mathbb{E}_{h_{00}, h_{i i}}\left[1-\exp \left\{-\iint_{A} \mu^{f} r d r d \phi\right\}\right] .
$$

where $\mu^{f}$ is the expected density of dominant interferers, $\mathrm{TX}_{i}$, for the packet at $\mathrm{RX}_{0}$. This is given as

$$
\begin{aligned}
\mu^{f}= & \mathbb{E}\left[\text { density of active dominant interferers for } \mathrm{RX}_{0}\right] \\
= & \operatorname{Pr}\left(\mathrm{TX}_{i} \text { placed at }(x, y)\right) \cdot \operatorname{Pr}\left(\mathrm{TX}_{i}-\mathrm{RX}_{i} \text { activated } \mid(x, y)\right) \\
& \quad \cdot \operatorname{Pr}\left(\mathrm{TX}_{i} \text { causes error at } \mathrm{RX}_{0} \mid \mathrm{TX}_{i} \text { active at }(x, y)\right) \\
= & \lambda\left(1-e^{-\frac{h_{i i} R^{-\alpha} r^{\alpha}}{\beta_{t}}}\right)\left(1-e^{-\frac{h_{i i} R^{-\alpha} r^{\alpha}}{\beta_{r}}}\right) e^{-\frac{h_{00} R^{-\alpha} r^{\alpha}}{\beta}} \cdot
\end{aligned}
$$

\subsection{Outage probability in fading networks}

Based on the derivations given above, we arrive at the following theorem.

Theorem 2. The outage probability of CSMA $A_{T X R X}$ in the presence of Rayleigh fading with varying sensing thresholds is given by

$$
P_{\text {out }}\left(\text { CSMA }_{\text {TXRX }}\right)=\bar{P}_{b}^{M}+\left(1-\bar{P}_{b}^{M}\right) \bar{P}_{r t 1} \bar{P}_{r t}^{N},
$$

where:

- $\bar{P}_{b} \approx \bar{P}_{b 1}\left(\beta_{t}\right)+\left(1-\bar{P}_{b 1}\left(\beta_{t}\right)\right) \bar{P}_{b 1}\left(\beta_{r}\right) \frac{\pi s_{r}^{f^{2}}-A_{o l}\left(s_{t}^{f}, s_{r}^{f}\right)}{\pi s_{r}^{f^{2}}}$ is the average backoff probability, where $\bar{P}_{b 1}\left(\beta_{t}\right)$ is approximated by the solution to

$\bar{P}_{b 1}\left(\beta_{t}\right)=1-e^{-\lambda\left[1-\bar{P}_{b}^{M}+\left(1-\bar{P}_{b}^{M}\right) \bar{P}_{r t 1} \frac{1-\bar{P}_{r t}^{N}}{1-\bar{P}_{r t}}\right] \pi R^{2} \beta_{t}^{2 / \alpha} \frac{2 \pi / \alpha}{\sin (2 \pi / \alpha)},}$

- $\bar{P}_{r t}=\mathbb{E}_{h_{00}}\left[\bar{P}_{r x}+\left(1-\bar{P}_{r x}\right) P_{\text {during }}\left(h_{00}\right)\right]$ is the probability that a packet is received in error in a retransmission attempt. $\bar{P}_{r x}$ is the average probability that the packet is in error at the start of each of its retransmissions, approximated by

$\bar{P}_{r x}=1-e^{-\lambda\left[1-\bar{P}_{b}^{M}+\left(1-\bar{P}_{b}^{M}\right) \bar{P}_{r t 1} \frac{1-\bar{P}_{r t}^{N}}{1-\bar{P}_{r t}}\right] \pi R^{2} \beta^{2 / \alpha} \frac{2 \pi / \alpha}{\sin (2 \pi / \alpha)}}$,

- $P_{\text {during }}\left(h_{00}\right)$ is the probability that an error occurs at some $t \in(0, T)$, approximated by

$$
\begin{aligned}
\tilde{P}_{\text {during }}\left(h_{00}\right)= & 1-e^{-} \lambda_{\text {active }}\left[\int_{0}^{\infty} 2 \pi\left(1-e^{-\frac{\mathbb{E}\left[h_{i i}\right] R^{-\alpha} r^{\alpha}}{\beta_{t}}}\right)\right. \\
& \left.\times\left(1-e^{-\frac{\mathbb{E}\left[h_{i i}\right] R^{-\alpha} r_{r}^{\alpha}}{\beta_{r}}}\right) e^{-\frac{h_{00} R^{-\alpha} r^{\alpha}}{\beta}} r d r\right] .
\end{aligned}
$$

- $\bar{P}_{r t 1}=\mathbb{E}_{h_{00}}\left[P_{r x \mid \text { active }}\left(h_{00}\right)+\left(1-P_{r x \mid \text { active }}\left(h_{00}\right)\right)\right.$ $\left.P_{\text {during }}\left(h_{00}\right)\right]$ is the probability that the packet is received in error at its first transmission attempt, with $P_{r x \mid \text { active }}\left(h_{00}\right)$ approximated by

$\tilde{P}_{r x \mid \text { transmit }}\left(h_{00}\right)=\mathbb{E}_{h_{0 i}}\left[\bar{P}_{r x} \frac{A_{r x \mid \text { active }}\left(s_{t}^{f}\left(h_{00}, h_{0 i}\right), s_{r}^{f}\left(h_{00}, h_{0 i}\right)\right)}{\pi s^{f}\left(h_{00}, h_{0 i}\right)^{2}}\right]$,

where $s_{t}^{f}\left(h_{00}, h_{0 i}\right)$ is given by Equation (18). 
Optimization of the sensing thresholds is carried out in the next section, and comparison between CSMA $\mathrm{TXRX}_{\mathrm{TX}}$ with the other CSMA versions is performed in Section 6.

\section{Optimizing the sensing thresholds}

In order to find the optimal sensing thresholds of CSMA $_{\text {TXRX }}, \beta_{t}^{\text {opt }}$, and $\beta_{r}^{\text {opt }}$, such that the outage probability is minimized, we must in principle differentiate the outage probability expressions given in Theorems 1 and 2 with respect to $s_{t}$ and $s_{r}$, and set each equal to 0 . However, because of the complexity of our equations, this turns out to be a nontrivial task. Hence, we attack our optimization problem from another angle; we evaluate the total outage probability of CSMA $A_{\text {TXRX }}$ based on the change in the exposed and hidden node problems.

The hidden node problem of CSMA occurs during an active packet transmission when a newly arriving transmitter, $\mathrm{TX}_{i}$, is located too close to the receiver under observation, $\mathrm{RX}_{0}$, while $\mathrm{TX}_{i}$ and $\mathrm{RX}_{i}$ are simultaneously too far away from $\mathrm{TX}_{0}$ to detect its transmission. That is, $\mathrm{TX}_{i}$ initiates its transmission and causes outage for $\mathrm{RX}_{0}$ because $\mathrm{TX}_{0}$ is hidden to it and its receiver. The probability of such an event occurring is

$\operatorname{Pr}($ hidden node $) \approx \operatorname{Pr}\left(\mathrm{RX}_{0}\right.$ mid. $\left.\mid \overline{\mathrm{TX}_{i} \text { beg. } \cap \mathrm{RX}_{i} \text { beg. }}\right)$.

The exposed node problem occurs when a packet transmission is backed off even though its transmission would not have contributed to any outages. This is the case when the interferer $\mathrm{TX}_{i}$ or its receiver $\mathrm{RX}_{i}$ are located too close to the active transmission of $\mathrm{TX}_{0}$, but far enough from $\mathrm{RX}_{0}$ to not cause any errors for it. That is,

$\operatorname{Pr}($ exposed node $) \approx \operatorname{Pr}\left(\mathrm{TX}_{i}\right.$ beg. $\cup \mathrm{RX}_{i}$ beg. $\left.\mid \overline{\mathrm{RX}_{0} \text { mid. }}\right)$.

The exposed node problem is a direct consequence of the transmitter making the backoff decision.

\subsection{Optimization in the absence of fading}

In a non-fading network, we note that $P_{\text {out }}\left(\right.$ CSMA $\left._{\text {TXRX }}\right)$ is a convex function of $s_{t}$ and $s_{r}$ for low densities. As a simplified proof for this claim, we note that the total outage probability of CSMA $\mathrm{TXRX}_{\text {may for high densities }}$ be approximated by the summation of error probability expressions, which are of the form $P_{\text {error }}=1-e^{-\lambda_{\mathrm{csma}} \pi s^{2}}$. Differentiating this expression twice with respect to $s=$ $\left\{s_{t}, s_{r}\right\}$ yields

$$
\frac{d^{2} P_{\text {error }}}{d s^{2}}=2 \pi \lambda_{\text {csma }} e^{-\pi \lambda_{\text {csma }} s^{2}}\left(1-2 \pi \lambda_{\text {csma }} s^{2}\right) .
$$

For $2 \pi \lambda_{\text {csma }} s^{2}<1$, we have that $\frac{d^{2} P_{\text {error }}}{d s^{2}}>0$, indicating convexity. Hence, we may conclude that for low enough values of the density (where our approximate expressions are more accurate), $P_{\text {error }}$ (and thereby $P_{\text {out }}\left(\right.$ CSMA $\left._{\text {TXRX }}\right)$ ) is a convex function of $s$.

This means that in order to obtain $\beta_{t}^{\text {opt }}$ and $\beta_{r}^{\text {opt }}$ (equivalently $s_{t}^{\text {opt }}$ and $s_{r}^{\text {opt }}$ ), we may minimize the outage probability with respect to each variable separately. Starting from Equation (9), we have that the derivative of the outage probability for arbitrary values of $M$ and $N$ with respect to the sensing radius $s_{t}$ (equivalently $s_{r}$ ) is

$$
\begin{aligned}
\frac{d P_{\text {out }}\left(\text { CSMA }_{\text {TXRX }}\right)}{d s_{t}}= & M P_{b}^{M-1}\left(1-P_{r t}^{N+1}\right) \frac{d P_{b}}{d s_{t}} \\
& +(N+1) P_{r t}^{N}\left(1-P_{b}^{M}\right)\left(1-P_{r x}\right) \\
& \times \frac{d P_{\text {during }}}{d s_{t}},
\end{aligned}
$$

where $P_{r t}=P_{r x}+\left(1-P_{r x}\right) P_{\text {during. }}$. The optimal value for $s_{t}$ is then the solution to $\frac{d P_{\text {out }}\left(\mathrm{CSMA}_{\mathrm{TXRX}}\right)}{d s_{t}}=0$, which must be solved numerically.

In order to find a closed-form expression for the optimal values of $s_{t}$ and $s_{r}$, we consider the particular case of $M=$ 1 and $N=0$. This yields

$$
P_{\text {out }}\left(\text { CSMA }_{\text {TXRX }}\right)= \begin{cases}P_{r x}+\left(1-P_{r x}\right) P_{\text {during }} & ; s_{r}<s \\ P_{b}+\left(1-P_{b}\right) P_{\text {during }} & ; \text { otherwise }\end{cases}
$$

First set $s_{t}$ to be a fixed value (e.g., $s_{t}=0$ as in [12]). We know from [12] that the optimal $s_{r}$ that minimizes the outage probability of CSMA $\mathrm{RX}$ is $s_{r}^{\mathrm{opt}}=s$, which corresponds to $\beta_{r}^{\text {opt }}=\beta$. The intuition behind this is as follows:

- For $s_{r}<s \Rightarrow B\left(\mathrm{RX}_{0}, s_{r}\right)<B\left(\mathrm{RX}_{0}, s\right) \Rightarrow$ lower probability of backoff $\Rightarrow$ higher probability that outage occurs during an active transmission. Note that the reduction in the backoff probability does in fact not result in a reduction in the probability that outage occurs during a transmission, because even though $B\left(\mathrm{RX}_{0}, s_{r}\right)<B\left(\mathrm{RX}_{0}, s\right)$, any active transmissions inside $B\left(\mathrm{RX}_{0}, s\right)$ upon the arrival of $\mathrm{TX}_{0}-\mathrm{RX}_{0}$ will contribute to the outage. Hence, if $s_{r}<s$, the total outage probability will be higher than its minimum value.

- For $s_{r}>s \Rightarrow B\left(\mathrm{RX}_{0}, s_{r}\right)>B\left(\mathrm{RX}_{0}, s\right) \Rightarrow$ higher probability of backoff $\Rightarrow$ lower probability that outage occurs during an active transmission. However, this decrease is less than the increase in the backoff probability, because the change in the area of $B\left(\mathrm{RX}_{0}, s_{r}\right)$ is larger than the decrease in the 
circumference of the circle around $\mathrm{TX}_{i}$ where $\mathrm{RX}_{i}$ can be located. Hence, the total outage probability increases as $s_{r}$ increases beyond $s$.

Assuming that $s_{r}^{\text {opt }}=s$, we have that $P_{\text {out }}\left(\right.$ CSMA $\left._{\text {TXRX }}\right)$ is a function of $s_{t}$ only. In order to find the optimal sensing radius of the transmitter, $s_{t}{ }^{\text {opt }}$, we use the outage probability of $\mathrm{CSMA}_{\mathrm{RX}}{ }^{\mathrm{a}}$ as a reference. The probability that $\mathrm{TX}_{0}$ is hidden to both $\mathrm{TX}_{i}$ and $\mathrm{RX}_{i}$ is lower than the probability that it is hidden only to $\mathrm{RX}_{i}$, which is the case in $\mathrm{CSMA}_{R X}$. Hence, the transmitter sensing of CSMA $\mathrm{TXRX}$ reduces the hidden node problem, as the area around $\mathrm{RX}_{0}$ where the activation of an interferer will and cause outage, is reduced. For a non-fading network, this yields the following approximation:

$$
\tilde{P}_{\text {during }} \approx \tilde{P}_{\text {during }}^{\mathrm{RX}}-\left(1-e^{-\lambda_{\text {csma }} A_{o l}\left(s_{t}, s_{r}\right)}\right),
$$

where $A_{o l}\left(s_{t}, s_{r}\right)$ is the area of overlap between $B\left(\mathrm{TX}_{0}, s_{t}\right)$ and $B\left(\mathrm{RX}_{0}, s_{r}\right)$, as illustrated in Figure 2 and given by Equation (6). The exposed node problem occurs when $\mathrm{TX}_{i}-\mathrm{RX}_{i}$ are located inside $B\left(\mathrm{TX}_{0}, s_{t}\right)$, but outside of $B\left(\mathrm{RX}_{0}, s\right)$. Compared to CSMA $\mathrm{RX}$, we have

$$
\tilde{P}_{b} \approx \tilde{P}_{b}^{\mathrm{RX}}+\left(1-e^{-\lambda_{\mathrm{csma}}\left(\pi s_{t}^{2}-A_{o l}\left(s_{t}, s_{r}\right)\right)}\right)
$$

where $\tilde{P}_{b}^{\mathrm{RX}}$ is the backoff probability of $\mathrm{CSMA}_{\mathrm{RX}}$ as derived in [12], and $A_{o l}\left(s_{t}, s_{r}\right)$ is given by Equation (6).

Now, we note that $\tilde{P}_{b}^{\mathrm{RX}}$ and $\tilde{P}_{\text {during }}^{\mathrm{RX}}$, which were derived in [12], are functions of $s$ and $s_{r}$ only (and not of $s_{t}$ ), which yields:

$$
\begin{aligned}
\frac{P_{\text {out }}\left(\mathrm{CSMA}_{\text {TXRX }}\right)}{d s_{t}}= & \left(1-P_{\text {during }}\right) \frac{d P_{b}}{d s_{t}}+\left(1-P_{b}\right) \frac{d P_{\text {during }}}{d s_{t}} \\
= & +\left(1-P_{\text {during }}\right) \lambda e^{-\lambda\left(\pi s_{t}^{2}-A_{o l}\left(s_{t}, s_{r}^{\mathrm{opt}}\right)\right)} \\
& \times \frac{d}{d s_{t}}\left(\pi s_{t}^{2}-A_{o l}\left(s_{t}, s_{r}^{\mathrm{opt}}\right)\right) \\
& -\left(1-P_{b}\right) \lambda e^{-\lambda A_{o l}\left(s_{t}, s_{r}^{\mathrm{opt}}\right)} \frac{d A_{o l}\left(s_{t}, s_{r}^{\mathrm{opt}}\right)}{d s_{t}} .
\end{aligned}
$$

Setting this derivative equal to 0 , we obtain:

$$
\begin{aligned}
& \left(1-P_{b}\right) e^{-\lambda A_{o l}\left(s_{t}, s_{r}^{\mathrm{opt}}\right)} \frac{d A_{o l}\left(s_{t}, s_{r}^{\mathrm{opt}}\right)}{d s_{t}} \\
& \left.-\left(1-P_{\text {during }}\right) e^{-\lambda\left(\pi s_{t}^{2}-A_{o l}\left(s_{t}, s_{r}^{\mathrm{opt}}\right)\right.}\right)\left(2 \pi s_{t}-\frac{d A_{o l}\left(s_{t}, s_{r}^{\mathrm{opt}}\right)}{d s_{t}}\right)=0 .
\end{aligned}
$$

We have that $\frac{d A_{o l}\left(s_{t}, s_{r}\right)}{d s_{t}}=2 s_{t} \cos ^{-1}\left(\frac{s_{t}}{2 R}\right)$ when $s_{r}=$ $s \approx R$. For $s \leq R$ (i.e., for small values of $\beta$ ), we have that $s_{t}^{\text {opt }}=0$, i.e., it is beneficial to have no transmitter sensing at all, as is the case in CSMA $\mathrm{RX}$. For $s>R, s_{t}^{\text {opt }}$ is found numerically as the nonzero solution to Equation (37), which is plotted in Figure 3 for $\beta=10 \mathrm{~dB}$. The reason we apply a high value for $\beta$ (compared to the value of $0 \mathrm{~dB}$ that has been applied before), is to emphasize the benefit of the transmitter sensing.

The point where the outage probability is decreasing at its highest rate, i.e., the minimum point of $\frac{d P_{\text {out }}\left(\mathrm{CSMA}_{\mathrm{TXRX}}\right)}{d s_{t}}$, occurs for $s_{t}=s-R^{\mathrm{b}}$. This corresponds to $\beta_{t}=\left(\beta^{1 / \alpha}-1\right)^{\alpha}$. For this value of $\beta_{t}, B\left(\mathrm{RX}_{0}, s\right)$ covers $B\left(\mathrm{TX}_{0}, s_{t}\right)$ completely, meaning that the transmitter sensing of CSMA $\mathrm{AXRX}_{\mathrm{TX}}$ introduces no additional exposed node problems, while at the same time providing some protection for its receiver, thus reducing the hidden node problem. That is, for $s>R, s_{t}$ can be increased up to $(s-R)$ without introducing any exposed node problems,

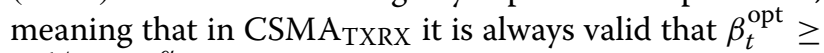
$\left(\beta^{1 / \alpha}-1\right)^{\alpha}$.

\subsection{Optimization in the presence of fading}

In the case of fading, the optimization problem becomes more complicated, as we can no longer translate it to a distance problem. Intuitively, we would expect CSMA ${ }_{\text {TXRX }}$ to yield an optimal performance when $\beta_{r}=\beta$ and $\beta_{t}=0$. The reason for this is as follows:

- If $\beta_{r}>\beta$, the exposed node problem is increased, while the hidden node problem is not reduced (this is partly because we do not consider the aggregate interference power in our derivations). On the other hand, if $\beta_{r}<\beta$, there is no exposed node problem, but the hidden node problem is higher than when $\beta_{r}=\beta$. Hence, $\beta_{r}^{\text {opt }}=\beta$.

- Next, we evaluate the benefit that the transmitter sensing of CSMA TXRX provides. Since the channel coefficient from $\mathrm{TX}_{i}$ to $\mathrm{TX}_{0}$ is independent from the channel coefficient from $\mathrm{TX}_{0}$ to $\mathrm{RX}_{i}$, the decision-making of the $\mathrm{TX}_{i}$ based on its own channel does not provide much benefit for the packet reception at its receiver (in terms of the hidden node problem). In fact, the transmitter's decision to back off from transmission when its receiver wishes to activate it, is only adding to the exposed node problem. Hence, $\beta_{t}^{\text {opt }}=0$.

In order to validate the reasoning given above, we evaluate the derivative of the outage probability in the presence of fading for $M=1$ and $N=0$;

$$
\begin{aligned}
\frac{d \bar{P}_{\text {out }}\left(\text { CSMA }_{\text {TXRX }}\right)}{d \beta_{t}} \approx & \left(1-\bar{P}_{\text {during }}\right) \frac{d \bar{P}_{b}}{d \beta_{t}} \\
& +\left(1-\bar{P}_{b}\right) \frac{d \bar{P}_{\text {during }}}{d \beta_{t}}=0 .
\end{aligned}
$$


First, we find the derivative of $\bar{P}_{\text {during; }}$;

$$
\begin{aligned}
\frac{d \bar{P}_{\text {during }}}{d \beta_{t}}= & 2 \pi \lambda \mathbb{E}_{h_{00}}\left[e^{-\lambda \int_{0}^{\infty} 2 \pi\left(1-e^{-\frac{\mathbb{E}\left[h_{i i}\right] R^{-\alpha} r^{\alpha}}{\beta_{t}}}\right)\left(1-e^{-\frac{\mathbb{E}\left[h_{i i}\right] R^{-\alpha} r^{\alpha}}{\beta r}}\right) e^{-\frac{h_{00} R^{-\alpha} r^{\alpha}}{\beta}} r d r}\right. \\
& \left.\times \int_{0}^{\infty}\left(1-e^{-\frac{\mathbb{E}\left[h_{i i}\right] R^{-\alpha} r^{\alpha}}{\beta_{t}}}\right) e^{-\frac{\mathbb{E}\left[h_{i i}\right] R^{-\alpha} r_{r}^{\alpha}}{\beta_{r}}} e^{-\frac{h_{00} R^{-\alpha} r_{r}^{\alpha}}{\beta}}\left(-\frac{\mathbb{E}\left[h_{i i}\right] R^{-\alpha} r^{\alpha}}{\beta_{t}^{2}}\right) r d r\right] .
\end{aligned}
$$

Differentiating the backoff probability, $\bar{P}_{b}$, given by Theorem 2, yields

$$
\begin{aligned}
\frac{d \bar{P}_{b}}{d \beta_{t}}= & \frac{d \bar{P}_{b 1}\left(\beta_{t}\right)}{d \beta_{t}} \\
& +\left(1-\bar{P}_{b 1}\left(\beta_{t}\right)\right) \bar{P}_{b 1}\left(\beta_{r}\right) \mathbb{E}_{h_{00}, h_{0 i}}\left[\frac{1}{\pi s_{r}^{f^{2}}} \frac{d A_{o l}\left(s_{t}^{f}, s_{r}^{f}\right)}{d \beta_{t}}\right] \\
& -\frac{d \bar{P}_{b 1}\left(\beta_{t}\right)}{d \beta_{t}} \bar{P}_{b 1}\left(\beta_{r}\right) \mathbb{E}_{h_{00}, h_{0 i}}\left[1-\frac{A_{o l}\left(s_{t}^{f}, s_{r}^{f}\right)}{\pi s_{r}^{2}}\right], \quad(40)
\end{aligned}
$$

where $A_{o l}\left(s_{t}^{f}, s_{r}^{f}\right)$ is given by Equation (6). Note that when $(M, N)=(1,0), \bar{P}_{b 1}\left(\beta_{t}\right)$ may be expressed by the Lambert function $\mathcal{W}_{0}(\cdot)$, as given below;

$$
\begin{aligned}
\bar{P}_{b 1}\left(\beta_{t}\right)= & 1-\frac{1}{\pi \lambda R^{2} \beta_{t}^{2 / \alpha} \frac{2 \pi / \alpha}{\sin (2 \pi / \alpha)}} \\
& \times \mathcal{W}_{0}\left(\pi \lambda R^{2} \beta_{t}^{2 / \alpha} \frac{2 \pi / \alpha}{\sin (2 \pi / \alpha)}\right) .
\end{aligned}
$$

Setting $x=\pi \lambda R^{2} \beta_{t}^{2 / \alpha} \frac{2 \pi / \alpha}{\sin (2 \pi / \alpha)}$, and differentiating $\bar{P}_{b 1}\left(\beta_{t}\right)$ yields

$$
\begin{aligned}
\frac{d \bar{P}_{b 1}\left(\beta_{t}\right)}{d \beta_{t}} & =\frac{\mathcal{W}_{0}(x)}{x^{2}}-\frac{1}{x} \cdot \frac{\mathcal{W}_{0}(x)}{x\left[1+\mathcal{W}_{0}(x)\right]} \\
& =\frac{\mathcal{W}_{0}(x)^{2}}{x^{2}\left[1+\mathcal{W}_{0}(x)\right]}
\end{aligned}
$$

Inserting these expressions back into Equation (38), the optimal value of $\beta_{t}$ can be found numerically. This is shown in Figure 4 , where $\frac{d P_{\text {out }}\left(\mathrm{CSMA}_{\mathrm{TXRX}}\right)}{d \beta_{t}}$ is plotted as a function of $\beta_{t}$ for various values of $\beta_{r}$. $\beta_{t}^{\text {opt }}$ is potentially the point where the derivative of $P_{\text {out }}$ (CSMA TXRX $_{\text {TX }}$ crosses 0 . As expected, $\frac{d P_{\text {out }}\left(\mathrm{CSMA}_{\mathrm{TXRX}}\right)}{d \beta_{t}}$ is positive for all $\beta_{t}$, meaning that it is always an increasing function of $\beta_{t}$. In other words, the outage probability of CSMA TXRX in the presence of fading is minimized for $\beta_{r}^{\text {opt }}=\beta$ and $\beta_{t}^{\text {opt }}=0$.

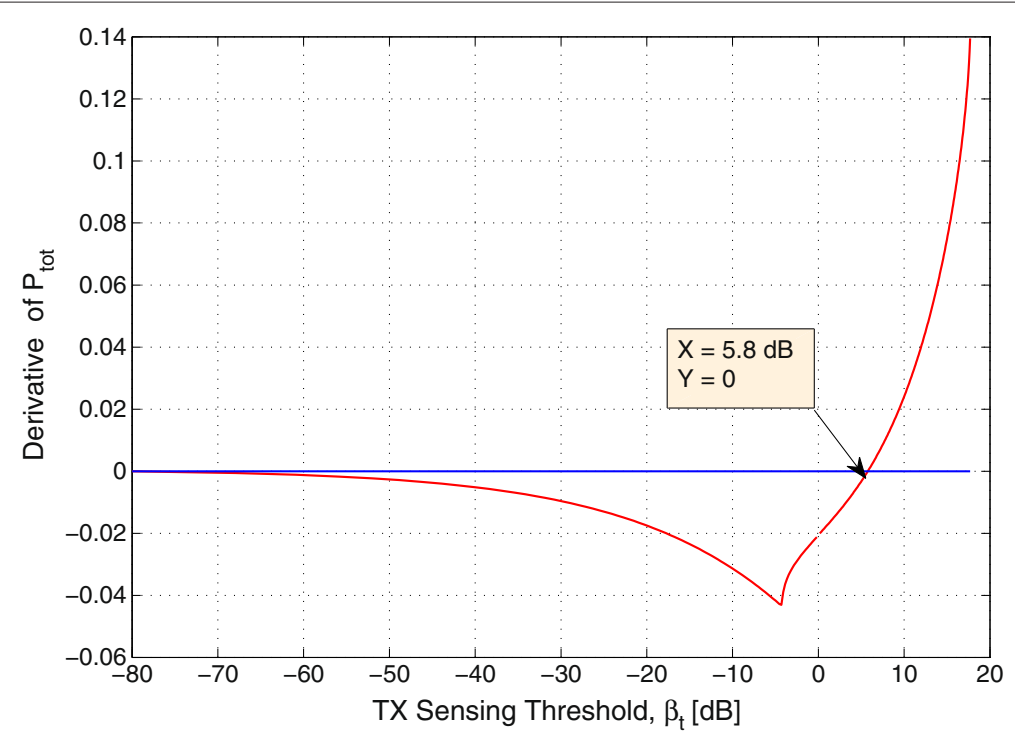

Figure 3 Derivative of the outage probability of CSMA TXRX for $\lambda=0.01, \beta=10 \mathrm{~dB}, M=1$, and $N=0$. 


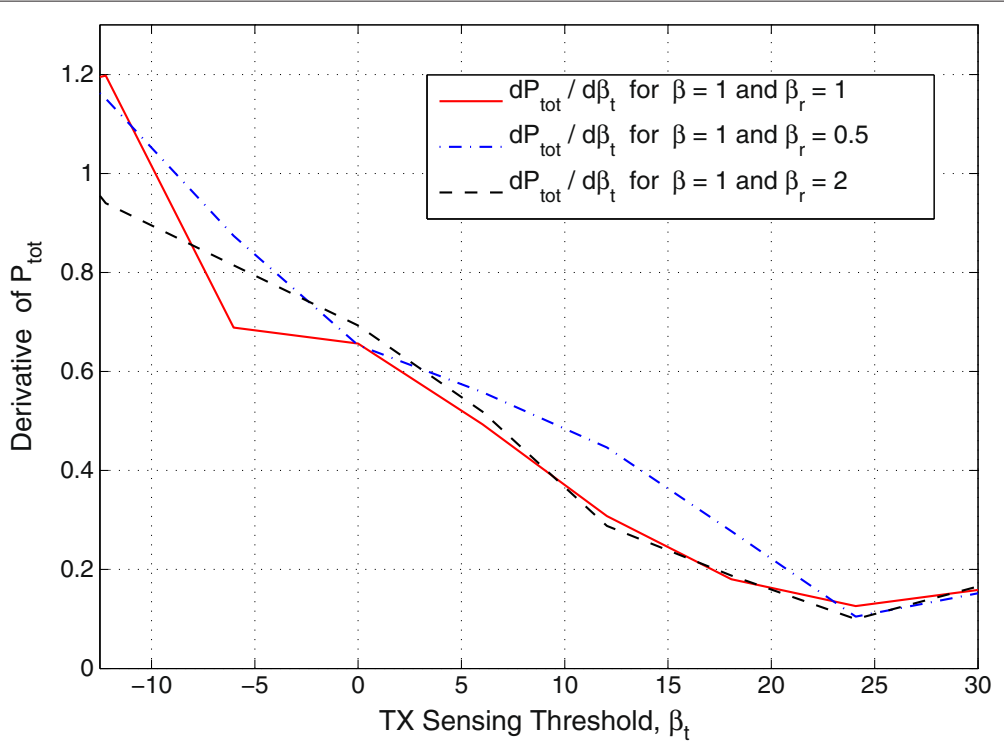

Figure 4 Derivative of the outage probability of CSMATXRX in a fading network as a function of $\beta_{t}$, for $\beta=0 \mathrm{~dB}$.

\section{Numerical results}

In Figures 5 and 6, the outage probability of CSMA TXRX (and $\mathrm{CSMA}_{\mathrm{RX}}$ for the sake of comparison) are plotted as a function of the packet arrival density $\lambda$, for a nonfading and a fading network, respectively. Firstly, our analytical expressions are validated as they follow the simulation results tightly. Note that in the presence of fading, some discrepancies can be observed between the analytical results and simulations; this is due to the additional source of randomness coming from the channel coefficients, making the "dominant interferer" approximation used in the derivations less accurate. However, these deviations occur at high densities corresponding to a high outage probability $(>0.3)$, which is at least an order of magnitude higher than what is often applied in practical networks.

Second, we observe that as the density increases, so does the outage probability, until the network reaches a point of saturation, where $P_{\text {out }} \approx 1$. By increasing the number of backoffs and retransmissions, significant

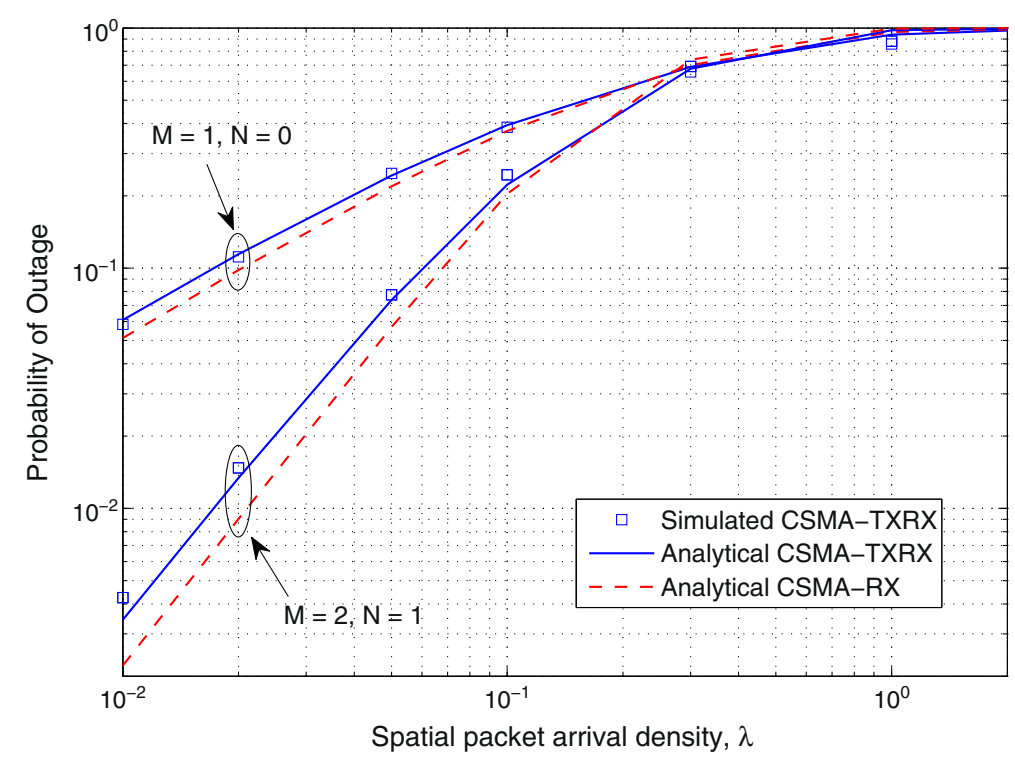

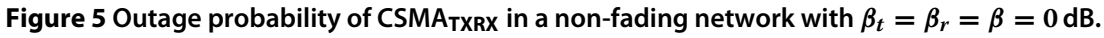




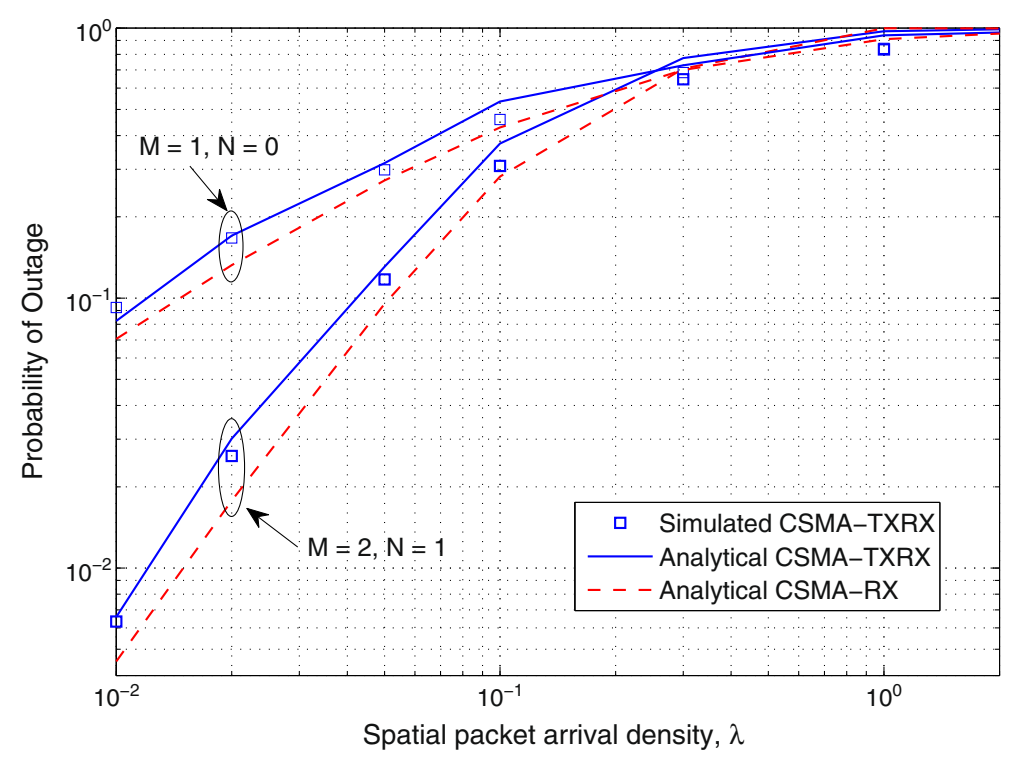

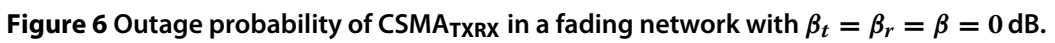

performance gain can be obtained. For low densities with $(M, N)=(2,1)$, the outage probability is up to 10 times lower than when $(M, N)=(1,0)$. Thirdly, the addition of transmitter sensing in CSMA TXRX $_{\text {does not appear to }}$ provide any improvement compared to CSMA $\mathrm{RX}_{\mathrm{RX}}$. In fact, CSMA $_{R X}$ outperforms CSMA TXRX $_{\text {by }}$ up to $20 \%$ in nonfading networks and up to $50 \%$ when fading is present. This is due to the exposed node problem caused by the

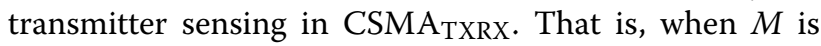
small, the protection that the transmitter sensing provides does not counterbalance the backoff probability increase it generates.
In Figure 7 , the outage probability of CSMA is plotted as a function of the sensing thresholds $\beta_{t}$ and $\beta_{r}$, for a fixed low density of $\lambda=0.01$, $\beta=10 \mathrm{~dB}$, and $(M, N)=(1,0)$. The plot shows that $\beta_{r}^{\text {opt }}=\beta=10$ and $\beta_{t}^{\text {opt }}=5.7 \mathrm{~dB}$, which is also obtained by numerically solving Equation (37), thus confirming our conclusions from Subsection 5.1. Similarly, in Figure 8 , the outage probability of CSMA $_{\text {TXRX }}$ is considered in a fading network with $\lambda=0.03$, $\beta=0 \mathrm{~dB}$, and $(M, N)=(1,0)$. Again, our derivations from Subsection 5.2 are confirmed, i.e., $\beta_{r}^{\text {opt }}=\beta=0 \mathrm{~dB}$ and $\beta_{t}^{\text {opt }}=0$.

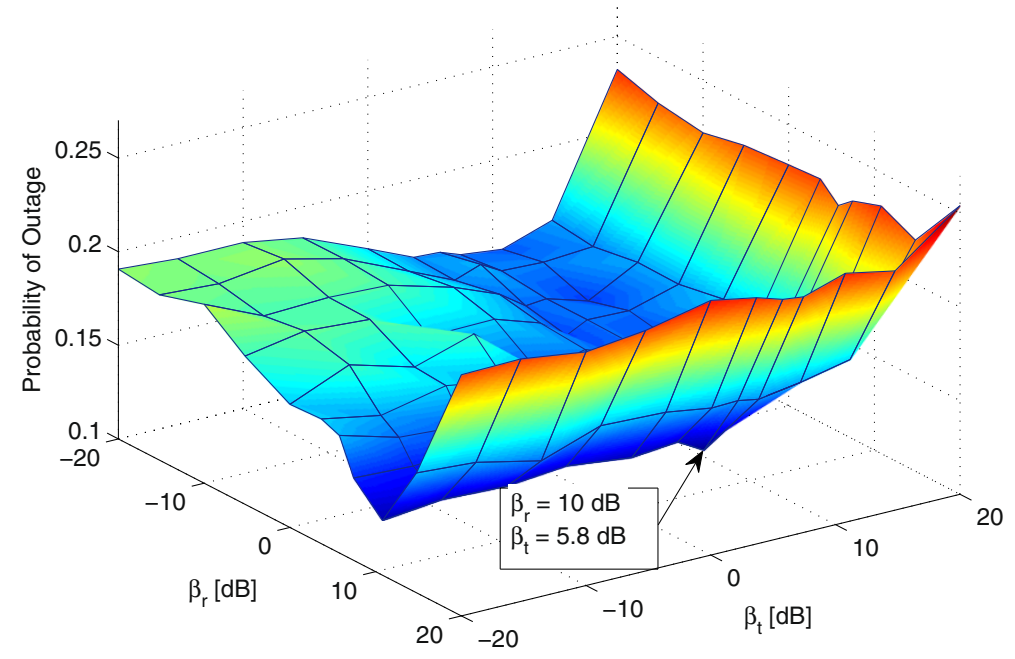

Figure 7 Outage probability of $\operatorname{CSMA}_{\mathrm{TXRX}}$ as a function of the sensing thresholds, for a non-fading network with $\lambda=0.01, \beta=10 \mathrm{~dB}$ and $(M, N)=(1,0)$ 


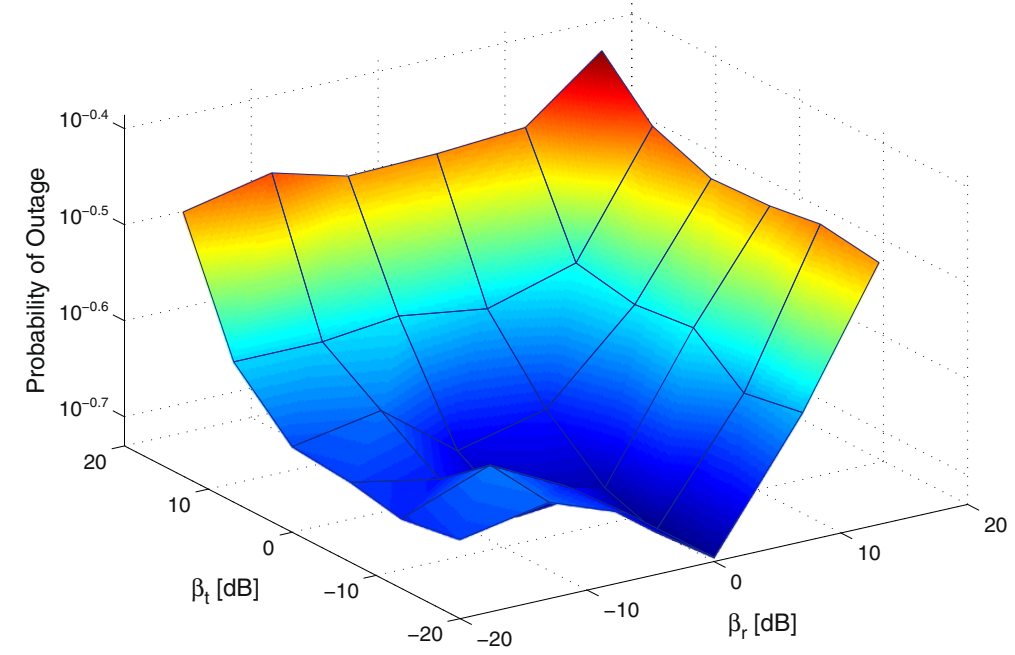

Figure 8 Outage probability of $\operatorname{CSMA}_{\mathrm{TXRX}}$ as a function of the sensing thresholds, for a fading network with $\lambda=0.03, \beta=0 \mathrm{~dB}$, and $(M, N)=(1,0)$.

The impact of the number of backoffs, $M$, is illustrated in Figure 9, where the receiver sensing threshold is assumed to be constant, $\beta_{r}=\beta=0 \mathrm{~dB}$, while $M$ and the transmitter sensing threshold $\beta_{t}$ are optimized jointly. As expected, the outage probability decreases monotonically with $M$. For each $M$, there is a different value for $\beta_{t}^{\text {opt }}$, although the range of this is very small. Hence, we conclude that the result of Equation (37) to find $s_{t}^{\text {opt }}$ analytically for $M=1$, can be applied as an approximation for greater values of $M$ as well. The fact that outage probability reduces monotonically as $M$ increases is reinforced in Figure 10, which has the same parameter values as Figure 9.
Figure 11 emphasizes the effect of $M$ on the outage probability of CSMA $A_{T X R X}$, as compared to CSMA TX $_{\text {and }}$ CSMA $_{\mathrm{RX}}$. In this plot, we set $N=0$ and a high density of $\lambda=0.1$ is chosen. When only $M=1$ channel sensing is allowed before the packet is dropped, CSMA TXRX exhibits up to $10 \%$ higher outage probability than $\operatorname{CSMA}_{R X}$ and up to $20 \%$ lower outage probability than CSMA $_{\mathrm{TX}}$. As $M$ increases, the benefit of the joint channel sensing of CSMA TXRX becomes more evident; for $M=4$,

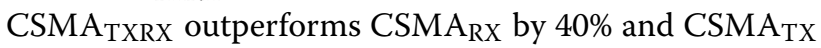
by a factor 2 . Hence, we conclude that by applying joint transmitter-receiver sensing in a network with $M>1$, the outage performance of CSMA can be improved beyond

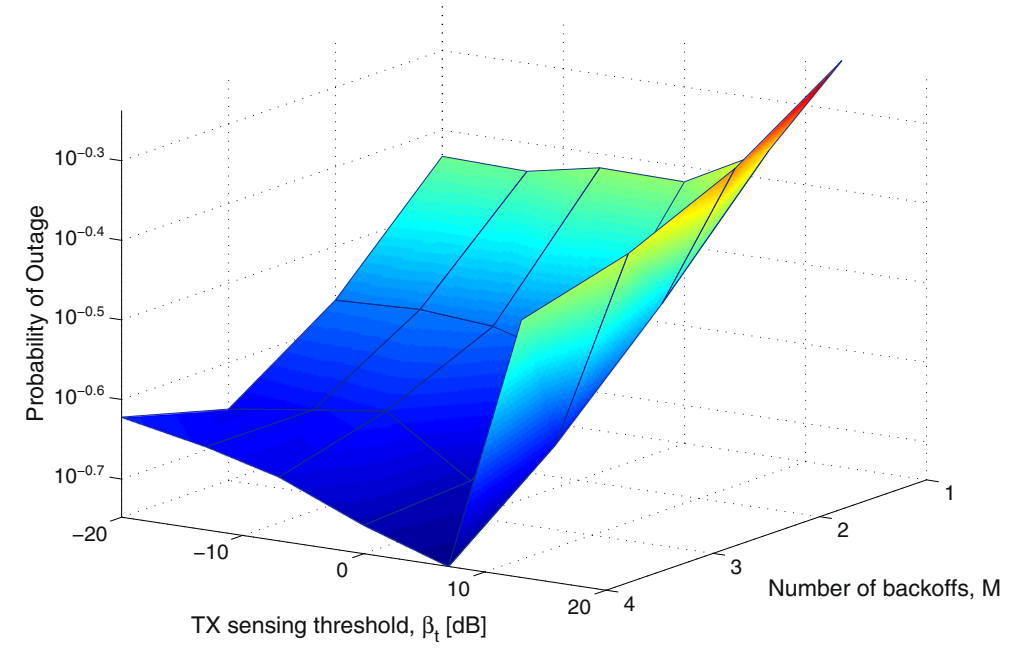

Figure 9 Outage probability of CSMATXRX as a function of $\beta_{t}$ and $M$, for a non-fading network with $\lambda=0.1, \beta=\beta_{r}=0 \mathrm{~dB}$, and $N=0$. 


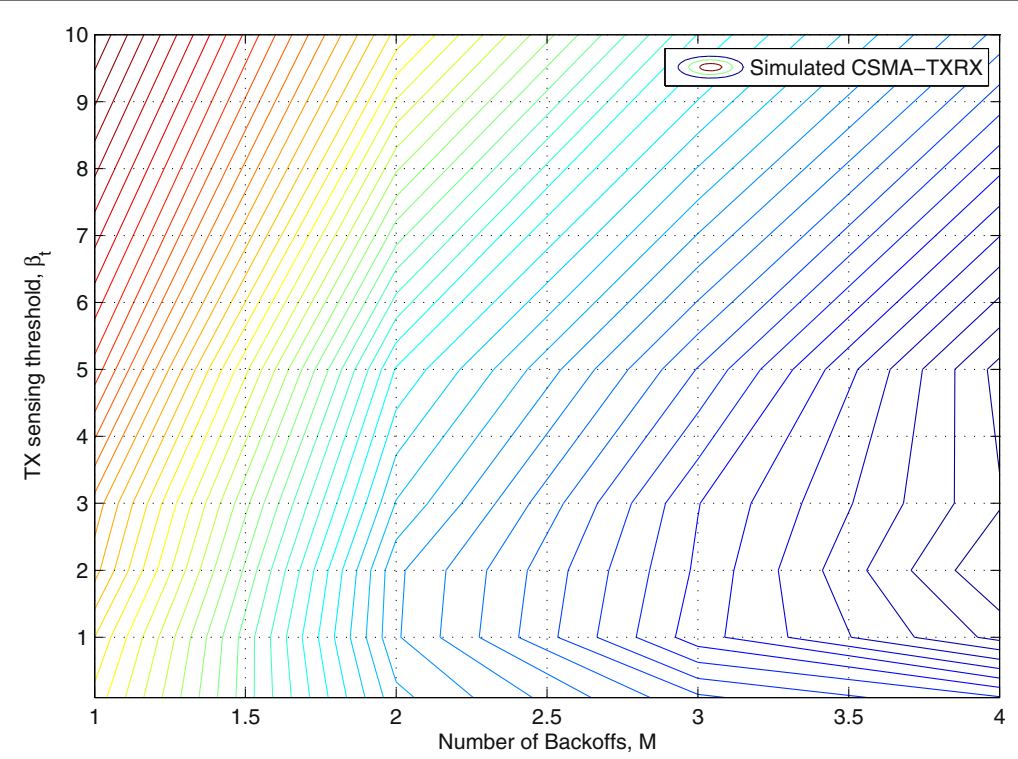

Figure 10 Contour plot of outage probability of CSMATXRX as a function of $\beta_{t}$ and $M$, for a non-fading network with $\lambda=0.1$, $\beta=\beta_{r}=0 \mathrm{~dB}$, and $N=0$.

that of CSMA $A_{R X}$. Moreover, the optimal sensing thresholds are $\beta_{r}^{\text {opt }}=\beta$ and $\beta_{t}^{\text {opt }}$ is found approximately as the solution to Equation (37).

The impact of retransmissions is opposite to that of backoffs. As seen from Figure 12, where the outage probability is plotted for a fixed density of $\lambda=0.03$ and $M=2$ backoffs, the outage probability of CSMA $\mathrm{RX}$

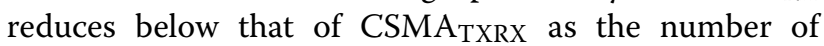
retransmissions, $N$, increases, e.g., when $(M, N)=(2,0)$, CSMA $_{\text {TXRX }}$ outperforms CSMA $A_{R X}$ by $10 \%$, while for
$(M, N)=(2,3)$, we have $20 \%$ higher outage probability for CSMA TXRX than for CSMA $_{R X}$. While significant gain is obtained by increasing $N$ from 0 to 2 , little benefit is observed for $N>2$. Note that we have included results on the impact of $M$ and $N$ only in non-fading networks, as similar conclusions are drawn in fading networks.

\section{Conclusions}

In this article, we improve the performance of CSMA in wireless ad hoc networks by introducing joint transmitter-

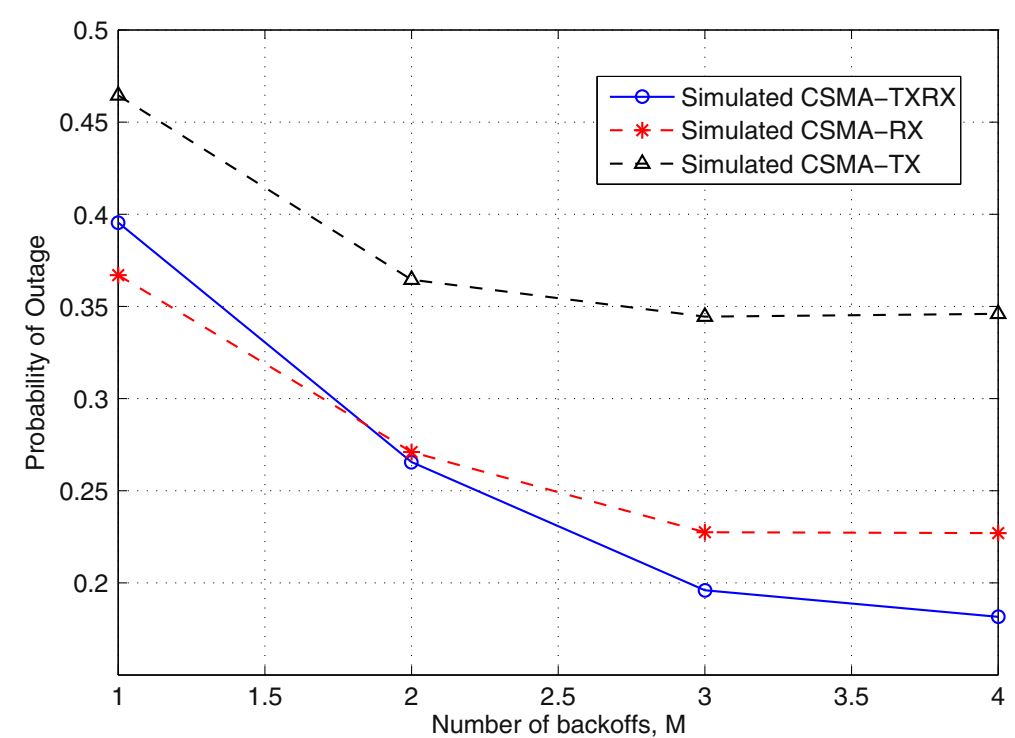

Figure 11 Outage probability of CSMA TXRX as a function of the number of backoffs, $M$, for a non-fading network with $\lambda=0.1$ and $N=0$. 


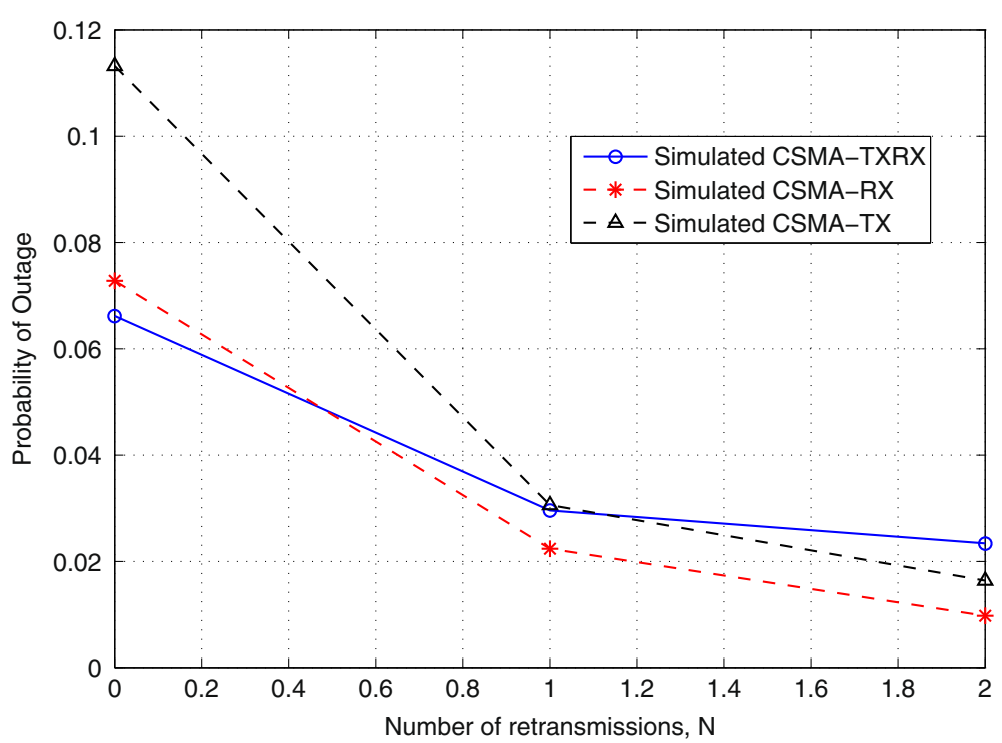

Figure 12 Outage probability of CSMA $\mathrm{TXRX}_{\text {as }}$ a function of the number of retransmissions, $N$, for a non-fading network with $\lambda=0.03$ and $M=2$.

receiver sensing and simultaneously optimizing the sensing thresholds of both the transmitter and the receiver.

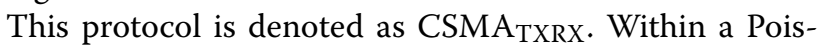
son distributed ad hoc network, approximate analytical expressions are derived for the outage probability of CSMA $_{\text {TXRX }}$ with respect to the transmission density and the sensing thresholds. The optimal sensing thresholds for both the transmitter and receiver are obtained both in non-fading and fading networks, and an understanding is provided for how these optimal thresholds balance between the hidden and exposed node problems of CSMA. It is shown that using optimal sensing thresholds can provide significant performance gain for all transmission densities. Moreover, when multiple backoffs are allowed, CSMA $A_{T X R X}$ outperforms CSMA $\mathrm{RX}_{\text {[12], which }}$ was previously shown to provide the best performance in unslotted systems, e.g., when $M=4$, this improvement is $40 \%$.

For future study, we wish to improve the performance of CSMA by investigating more efficient use and exchange of channel information between each transmitter and its receiver. Other possible extensions are to apply adaptive rate and power control to further improve the performance of CSMA in wireless ad hoc networks.

\section{Endnotes}

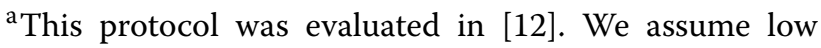
density of transmissions, where the outage probability expressions are good approximations.
${ }^{\mathrm{b}}$ This assumes that $s>R$, which is the case in most networks, as it ensures that the receiver can detect its own transmitter.

\section{Competing interest}

The authors declare that they have no competing interests.

Received: 1 August 2011 Accepted: 28 July 2012

Published: 23 August 2012

\section{References}

1. L Kleinrock, FA Tobagi, Packet switching in radio channels: Part I - Carrier sense multiple-access modes and their throughput-delay characteristics, IEEE Trans. Commun. 23, 1400-1416 (1975)

2. H Ma, R Vijaykumar, S Roy, J Zhu, Optimizing 802.11 wireless mesh networks performance using physical carrier sensing, IEEE/ACM Trans. Network. 17(5), 1550-1563 (2009)

3. Y Zhou, SM Nettles, in Proc. IEEE Wireless Communications and Networking Conference (WCNC), Balancing the hidden and exposed node problems with power control in csma/ca-based wireless networks, vol 2, (New Orleans, LA, 2005), pp. 683-688

4. G Ferrari, O Tonguz, in Proc. IEEE Military Communications Conference, MAC protocols and transport capacity in ad hoc wireless networks: Aloha versus PR-CSMA, vol 2, (Boston, MA, 2003), pp. 1113-1318

5. P Gupta, PR Kumar, The capacity of wireless networks, IEEE Trans. Inf. Theory. 46(2), 388-404 (2000)

6. LL Xie, PR Kumar, On the path-loss attenuation regime for positive cost and linear scaling of transport capacity in wireless networks, IEEE Trans. Inf. Theory. 52, 2313-2328 (2006)

7. JA Fuemmeler, NH Vaidya, W Veeravalli, in University of Illinois at Urbana-Champaign Technical Report Selecting transmit powers and carrier sense thresholds for CSMA protocols, 2004

8. BJB Fonseca, in Proc. Vehicular Technology Conference (VTC), A distributed procedure for carrier sensing threshold adaptation in CSMA-based mobile ad hoc networks, (Dublin, Ireland, 2007), pp. 66-70

9. J Zhu, X Guo, L Yang, W Conner, in Proc. IEEE International Conference on Communications (ICC), Leveraging spatial reuse in 802.11 mesh networks with enhanced physical carrier sensing, vol 7, (Budapest, Hungary, 2004), pp. 4004-4011 
10. A Hasan, JG Andrews, The guard zone in wireless ad hoc networks, IEEE Trans. Wirel. Commun. 6(3), 897-906 (2005)

11. S Weber, X Yang, J Andrews, G de-Veciana, Transmission capacity of wireless ad hoc networks with outage constraints, IEEE Trans. Inf. Theory. 51(12), 4091-4102 (2005)

12. M Kaynia, N Jindal, GE Øien, Performance analysis and improvement of MAC protocols in wireless ad hoc networks, IEEE Trans. Wirel. Commun. 10, 240-252 (2011)

13. KJ Park, L Kim, JC Hou, in Department of Computer Science UIUCDCS-R-2007-2884, University of Illinois at Urbana-Champaign Coordinating the interplay between physical carrier sense and power control in CSMA/CA wireless networks, 2007

14. H Ma, S Roy, in IEEE International Conference on Mobile Ad Hoc and Sensor Systems, MASS, Simple and effective carrier sensing adaptation for multi rate ad-hoc MESH networks, (Vancouver, Canada, 2006), pp. 795-800

15. F Rossetto, M Zorzi, in Proc. IEEE Global Communications Conference (GLOBECOM), Gaussian approximations for carrier sense modeling in wireless ad hoc networks, (Washington, DC, 2007), pp. 864-869

16. K Jeong, H Lim, in Proc. AMC CoNEXT, Experimental approach to adaptive carrier sensing in IEEE 802.15.4 wireless networks, (Madrid, Spain, 2008). article no. 47

17. P Muhlethaler, A Najid, in Proc. IEEE European Wireless Conference, Throughput optimization in multihop CSMA mobile ad hoc networks, (Barcelona, Spain, 2004)

18. J Zhu, B Metzler, X Guo, Y Liu, in Proc. IEEE INFOCOM, Adaptive CSMA for scalable network capacity in high-density WLAN: a hardware prototyping approach, (Barcelona, Spain, 2006, pp. 1-10

19. M Kaynia, GE Øien, N Jindal, in Proc. IEEE International Conference on Wireless Communications and Signal Processing (IC-WCSP), Joint transmitter and receiver carrier sensing capability of CSMA in MANETs, (Nanjing, China, (Best Paper Award), 2009), pp. 1-5

20. S Weber, J Andrews, N Jindal, The effect of fading, channel inversion, and threshold scheduling on ad hoc networks, IEEE Trans. Inf. Theory. 53(11), 4127-4149 (2007)

\section{doi:10.1186/1687-1499-2012-271}

Cite this article as: Kaynia and Øien: Optimizing the performance of nonfading and fading networks using CSMA with joint transmitter and receiver sensing. EURASIP Journal on Wireless Communications and Networking 2012 2012:271.

\section{Submit your manuscript to a SpringerOpen ${ }^{\circ}$ journal and benefit from:}

- Convenient online submission

- Rigorous peer review

- Immediate publication on acceptance

- Open access: articles freely available online

- High visibility within the field

- Retaining the copyright to your article

Submit your next manuscript at $\gg$ springeropen.com 